\title{
A SEMI-ANALYTIC MODEL OF TIDAL INLETS AND THEIR EVOLUTION
}

\author{
Magnus Larson, Lund University, magnus.larson@tvrl.Ith.se \\ Almir Nunes, Lund University, almir.nunes@tvrl.Ith.se \\ Hitoshi Tanaka, Tohoku University, hitoshi.tanaka.b7@tohoku.ac.jp \\ Hans Hanson, Lund University, hans.hanson@tvrl.Ith.se
}

\section{BACKGROUND}

The water exchange between the sea and a lagoon or bay through an inlet due to tides is a classical topic that has been investigated in a large number of studies (Keulegan, 1967; O'Brien and Dean, 1972; O'Brien and Clark, 1974; Escoffier, 1977). In this paper, a simple semi-analytic model of the flow induced by tides through an inlet connecting the sea to a lagoon or bay is developed. The model is employed to derive explicit expressions for key parameters associated with inlet flows such as bay water level amplitude, tidal prism, maximum inlet velocity, and mixing (retention) time. Also, the inlet flow model is combined with a sediment transport model to determine the conditions for equilibrium as well as the evolution of the inlet cross-sectional area towards equilibrium or closure.

\section{THEORY}

The flow through an inlet connecting a lagoon (or bay) to the sea was first described by Keulegan (1967) using the continuity and momentum equations resulting in,

$$
\frac{d \eta_{B}}{d t}=\frac{A_{l}}{A_{B}} \sqrt{\frac{2 g}{k_{l}}} \sqrt{\left|\eta_{o}-\eta_{B}\right|} \operatorname{sgn}\left(\eta_{o}-\eta_{B}\right)
$$

where $\eta_{B}$ is the bay water level, $\eta_{0}$ the sea water level (taken to be a sinusoidal), $A$ / the inlet cross-sectional area, $A_{B}$ the bay surface area, $g$ acceleration due to gravity, and $t$ time. The coefficient $k$, includes losses due to entrance, exit, and channel friction. Assuming $\eta в$ to be a sinusoidal at quasi-steady conditions with a certain amplitude reduction and phase shift compared to $\eta_{\mathrm{o}}$, the following approximate solution to Eq. 1 may be derived,

$$
\frac{a_{B}}{a_{o}}=\left(1-\frac{\left(C_{M} K\right)^{4}}{4}\left(\sqrt{1+\frac{4}{\left(C_{M} K\right)^{4}}-1}\right)^{2}\right)^{1 / 2}=\Psi(K)
$$

where $a_{B}$ and $a_{o}$ are the amplitudes in the ocean and bay, respectively, $K\left(=A, \sqrt{2 g a_{o}} T /\left(A_{B} 2 \pi a_{o} \sqrt{K_{l}}\right)\right.$, where $T$ is the period of the tide) the repletion coefficient (Keulegan, 1967), and $C_{M}$ an empirical fitting parameter. From Eqs. 1 and 2 the tidal prism, mean and maximum inlet velocity, and mixing time may be derived. Also, the semi-analytic solution can be used in a model for inlet evolution. Following Kraus (1998), the governing equation for temporal changes in $A_{\text {/ is }}$ given by,

$L \frac{d A_{l}}{d t}=\frac{k_{w}}{g}\left(u_{l}^{2}-u_{c r}^{2}\right) u, W-m_{L}$

where $L$ is the inlet length, $k_{w}$ an inlet transport coefficient, $u$ / the inlet velocity, $u_{c r}$ the critical velocity for transport, $W$ the inlet width, and $m_{L}$ the longshore transport rate. Eq. 3 may be written in non-dimensional form:

$$
\frac{d K}{d \hat{t}}=\left(\left(\frac{2}{\pi}\right)^{2}\left(\frac{\Psi(K)}{K}\right)^{2}-\hat{u}_{c r}^{2}\right) \frac{2}{\pi} \frac{\Psi(K)}{K} K^{n}-\hat{m}_{L}
$$

where $n$ is a power related to the inlet geometry (i.e., how $A_{\text {/ }}$ is related to $W$, see Larson et al., 2011) and ${ }^{\wedge}$ denotes a dimensionless variable.

\section{SELECTED RESULTS}

Fig. 1 illustrates how well the semi-analytic model (Eq. 2) reproduces the exact solution to Eq. 1 in terms of bay amplitude and mean velocity.

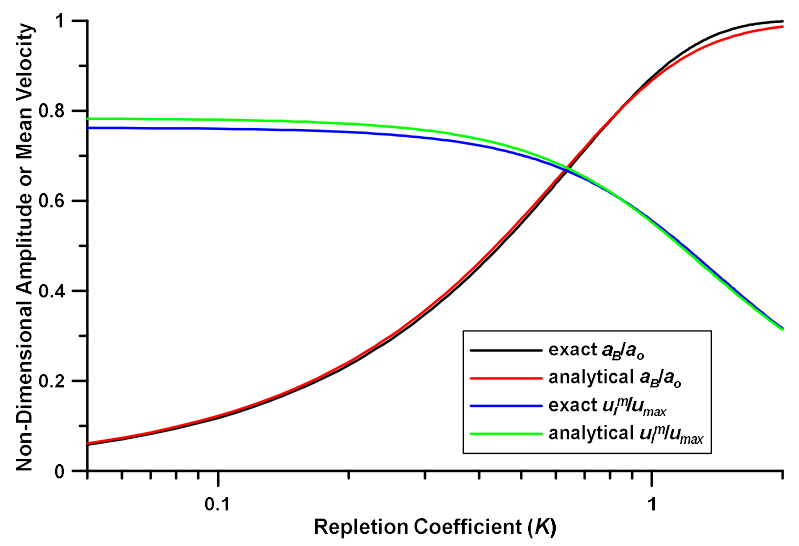

Figure 1 - Exact and approximate solution to inlet equation

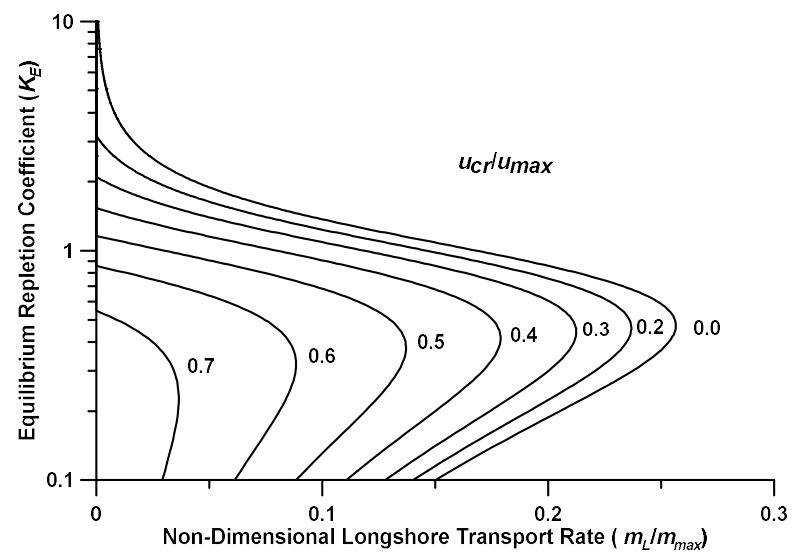

Figure 2 - Equilibrium conditions at an inlet

Fig. 2 displays the solution to Eq. 4 with $d K / d \hat{t}=0$, that is, for dynamic equilibrium conditions, yielding a curve similar to Escoffier (1940). For the results presented in Fig. 2 , a geometrical similar cross section was adopted $(n=1 / 2)$ In general, for a specific curve and a certain normalized longshore sediment transport (LST) rate, two solutions are obtained regarding the repletion coefficient at dynamic 
equilibrium $\left(K_{E}\right)$. The curves also present maximum LST rates at which no solution that involves a dynamic equilibrium is possible when exceeded.

Mota-Oliveira (1970), using a numerical model, calculated that for a repletion coefficient of about 0.6 to 0.8 the transport capacity reaches a maximum (assuming bed load to prevail). This is in agreement with the values presented in Fig. 2, especially at lower critical shear stresses. Seabergh (2003) demonstrated that a number of inlets in the United States had quite low K-values; in fact, such low values would indicate unstable inlets, if the Escoffier curve was analyzed, yet these inlets continue to stay open. The present analysis shows that the optimum $K$-value can be as low as what Seabergh (2003) noted, indicating that inlets can be stable for such values.

Illustrative model simulations with Eq. 4 towards equilibrium or closure due to perturbations of LST transport are presented in Fig. 3 .

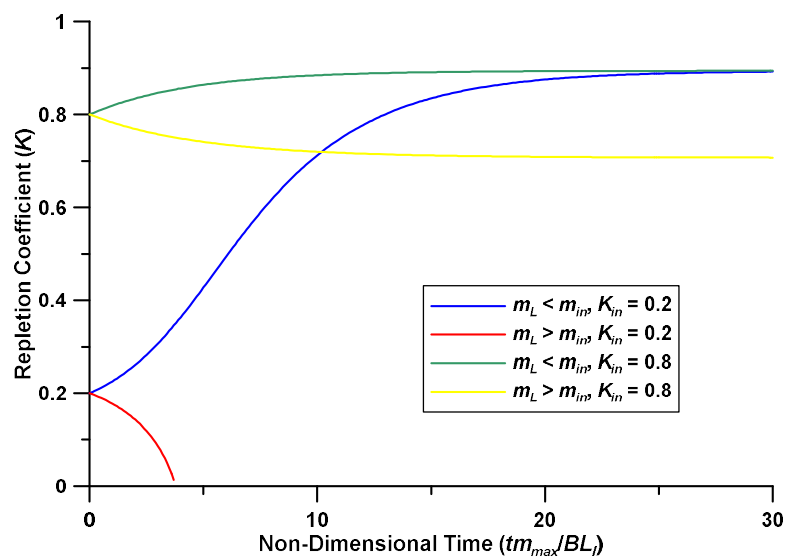

Figure 3 - Inlet area evolution in terms of the repletion coefficient for different non-dimensional sediment transport rates and initial repletion coefficient values.

In these simulations, the inlet was assumed to be in equilibrium initially $(\hat{t}=0)$, where the transport through the inlet $\hat{m}_{i n}=\hat{m}_{L}=0.19$ corresponding to the solutions $K_{E}=0.2$ and $K_{E}=0.8$ (see Fig. 2). Subsequently, $\hat{m}_{L}$ was increased to 0.21 or decreased to 0.17 , forcing the inlet towards a new equilibrium state. For $K_{E}<K_{E}^{\max }$ the equilibrium solution is not stable to small perturbations and either the inlet will close or the equilibrium for $K_{E}>K_{E}^{\max }$ will be attained. For $K_{\text {in }}=0.2$ an increase in the transport rate from $\hat{m}_{L}=0.19$ to 0.21 yields closure (red line), whereas a decrease from $\hat{m}_{L}=0.19$ to 0.17 moves the inlet towards a stable solution $K_{E}>K_{E}^{\max }$ corresponding to $\hat{m}_{L}=0.21$ (blue line). If a change in the transport rate occurs for $K_{\text {in }}=0.8$, increase or decrease, a new stable solution is found without any occurrence of inlet closure (green and yellow line).

\section{CONCLUDING REMARKS}

The semi-analytic model developed in this study reproduces several of the main features expected with regard to tidal inlet hydraulics and morphological evolution. Furthermore, the model made it possible to investigate the fundamental behavior of an inlet, yielding analytic expressions in non-dimensional form for key parameters such as bay amplitude, maximum and mean inlet velocity, tidal prism, and retention time. A self-similar shape was employed to describe the cross-sectional area, relating the depth and width of the inlet; this approach yields two solutions for equilibrium conditions (cf., Escoffier, 1977). Another interesting aspect of the stability analysis is that for a specific critical shear stress, there is an optimum repletion coefficient corresponding to a maximum normalized transport rate.

Asymptotic and equilibrium behavior could be established with the model. However, the next step is to investigate the ability of the model to simulate the inlet evolution under more realistic condition. A numerical approach will then be taken and comparisons with field data will be performed.

\section{ACKNOWLEDGEMENTS}

ML likes to acknowledge the support by the Japan Society for the Promotion of Science for a research visit to Tohoku University. AN carried out his part of the work funded by a scholarship from the CAPES Foundation within the Ministry of Education of Brazil, process $n^{\circ}$ 99999.002154/2015-03.

\section{REFERENCES}

Escoffier (1940): The stability of tidal inlets, Shore and Beach, 8(4), 114-115.

Escoffier (1977): Hydraulics and stability of tidal inlets, GITI Report 13, US Army Corps of Engineers, Waterways Experiment Station, Vicksburg, MS

Keulegan (1967): Tidal flow in entrances, Water-level fluctuations of basins in communication with seas, Technical Bulletin No. 14, US Army Corps of Engineers, Vicksburg, MS

Kraus (1998): Inlet cross-sectional area calculated by process-based model, Proceedings $26^{\text {th }}$ International Coastal Engineering Conference, ASCE, 3265-3278.

Larson, Hanson, Kraus and Hoan (2011): Analytical model for the evolution of coastal inlet cross-sectional area, Proceedings of Coastal Sediments '11, World Scientific, 444-457.

Mota-Oliveira (1970): Natural flushing ability in tidal inlets, Proceedings of the 12th Coastal Engineering Conference, ASCE, 1827-1845.

O'Brien and Clark (1974): Hydraulic constants of tidal entrances, Proceedings of the $14^{\text {th }}$ Coastal Engineering Conference, ASCE, 1546-1565.

O'Brien and Dean (1972): Hydraulics and sedimentary stability of tidal inlets, Proceedings of the $13^{\text {th }}$ International Coastal Engineering Conference, ASCE, 761-780.

Seabergh (2003): Long-term coastal inlet channel area stability, Proceedings Coastal Sediments '03, World Scientific (on CD). 